\title{
Pharmacokinetic and pharmacodynamic study of a phospholipid-based phase separation gel for once a month administration of octreotide
}

Mengxin Wang, Fengyin San, Yang Zou, Xun Sun, Zhi-Rong Zhang, Yao Fu*, Tao Gong*

Key Laboratory of Drug Targeting and Drug Delivery System, Ministry of Education, West China

School of Pharmacy, Sichuan University, Chengdu 610041, PR China

*Correspondence: Yao Fu, Tao Gong.

Key Laboratory of Drug Targeting, Ministry of Education, Sichuan University

No. 17. Section 3. Southern Renmin Road, Chengdu 610041 China.

Email: yfu4@scu.edu.cn (Y. Fu); gongtaoy@126.com (T. Gong)

Tel: +862885503798 (Y. Fu); +862885501615 (T. Gong) 


\begin{abstract}
As a natural somatostatin analog, octreotide acetate (OCT) has been extensively used in cancer treatment and growth hormone related diseases. The clinical application of OCT, however, is greatly limited by its short half-life, rapid elimination and clearance in vivo. In the current study, a high content phospholipid-based phase separation gel platform (PPSG) was presented, which could be injected in the soluble state and underwent rapid phase-separation into a gel-like implant after a single subcutaneous injection. OCT was dispersed homogeneously in the PPSG pre-gel solution to afford OCT-loaded PPSG (OCT-PPSG) after a single subcutaneous injection, which displayed controlled and sustained release profiles for up to $30 \mathrm{~d}$ in rats, rabbits and Beagle dogs. OCT-PPSG showed a less significant burst phase followed by a steady plasma concentration of OCT compared with Sandostatin ${ }^{\circledR}$ (LAR) in Beagle dogs. Moreover, OCT-PPSG was demonstrated to show remarkable antitumor efficacy in both a primary rat model and a xenograft mouse model of hepatocellular carcinoma (HCC). PPSG thus represented a promising and viable in situ forming gel platform material for the long-term sustained release of peptides and protein drugs.
\end{abstract}

Keywords: octreotide; phospholipid; in situ gel; sustained release; pharmacokinetics; hepatocellular carcinoma 


\section{Introduction}

With rapid advances in molecular biology and immunology, the number of biotech drugs, including peptides, proteins and monoclonal antibodies available for clinical use has dramatically increased in recent years [1]. Despite great therapeutic effects, most peptide and protein drugs are not stable under physiological conditions, which easily undergo degradation and are subjected to short in vivo half-life. Moreover, peptide and protein drugs display poor permeability across epithelial barriers and are thus commonly administered via parenteral injections that are not patient-friendly [2]. Non-parenteral routes of administration have been sought with varying degrees of success including oral [3-6], buccal [7], intranasal [8, 9], pulmonary [10-13], transdermal [14], ocular [15-17] and rectal [18-20] routes. Nevertheless, low bioavailability has remained the greatest challenge for non-parenteral routes. As a result, injectable peptide and protein formulations with extended release profiles and long-term therapeutic effect have been a topic of broad interests among pharmaceutical community. Long-acting injectables appear to overcome the biological barriers presented by non-parenteral routes, resulted in greater bioavailability. Meanwhile, a less frequent dosing regimen will likely help improve patient adherence.

Recently, our laboratory has successfully developed an injectable phospholipid-based phase separation gel (PPSG) to achieve either local or systemic delivery of long-acting therapeutics [21]. Using phosphatidylcholine, injection-grade oil and anhydrous ethanol as basic excipients, PPSG can be produced under hygienically-controlled conditions to achieve sustained and controlled release of various therapeutics. The PPSG gel platform materials remained in the soluble state before injection at room temperature and underwent rapid phase separation after subcutaneous injection to achieve transition into a gel-like material. After that, PPSG gel acted as a drug depot to release drugs in a controlled and sustained manner to achieve long acting therapeutic effects.

In the current study, the natural somatostatin analog octreotide was selected as the model peptide drug. Octreotide acetate (OCT) is much more potent and metabolically more stable than the parent drug somatostatin [22]. OCT has been reported to inhibit the secretion of growth hormones, insulin, glucagon [23] and gland secretion [24-26], which has thus become the treatment for acromegaly, carcinoid syndrome and endocrine tumors of the GI tract [27]. Besides, OCT displays high binding affinity for the somatostatin receptor 2 (SSTR2) and 5 (SSTR5) [28], and has demonstrated antitumor effects in hepatocellular carcinomas (HCC) with over expression of high-affinity SSTRs [29]. As a result, OCT is recommended as the primary treatment option when the probability of a surgical cure remains low [30]. In addition, OCT can be used as adjuvants for postoperative and preoperative therapy, and disease control between the administration and the onset of radiotherapy [30]. However, OCT has an in vivo half-life of less than $2 \mathrm{~h}$, and the therapeutic effect drops rapidly following subcutaneous injection or cessation of infusion [25]. To improve the therapeutic performance of OCT, two long-acting OCT formulations are currently available on the market: Lanreotide Autogel (Somatuline ${ }^{\circledR}$ Autogel/Depot; Ipsen Pharma, Paris, France) and Octreotide Long-acting Release (LAR) (Sandostatin ${ }^{\circledR}$ LAR; Novartis, Basel, Switzerland) [31]. However, the costs of manufacture and treatment for the two formulations are exceedingly high, which greatly increased the economic burden of the healthcare system.

Here, we introduced an OCT loaded PPSG (OCT-PPSG) and systematically investigated 
the in vitro release of OCT under varying release conditions. An important question needed to be addressed is whether consistent, extended and controlled release of OCT could be observed in different animal species. Thus we compared the pharmacokinetic profiles of OCT-PPSG via subcutaneous injection in rats, rabbits and Beagle dogs. Through in vitro-in vivo correlation (IVIVC) study of octreotide acetate solution (OCT-soln) and OCT-PPSG in three species, we sought to understand the mechanisms and correlation behind the in vitro release and in vivo absorption. Furthermore, the antitumor effect of OCT-PPSG was preliminarily investigated using both a primary rat model and a xenograft mouse model of hepatocellular carcinoma.

\section{Materials and methods}

\subsection{Materials}

Octreotide (OCT) and Sandostatin ${ }^{\circledR}$ LAR were donated by Capgemini biological pharmaceutical Ltd. (Chengdu, China). Phosphatidylcholine (E80) was purchased from Lipoid (Ludwigshafen, Germany). Injection-grade soybean oil was purchased from Tieling north Asia medicated oil Ltd. (Tieling, China). Heparin sodium was purchased from Biosharp biological pharmaceutical Ltd. (Shanghai, China). Acetonitrile, methanol, and water were all of HPLC grade and were purchased from Sigma-Aldrich (St Louis, USA). All other chemicals and solvents used were of analytical grade.

\subsection{Animals}

Healthy male Sprague-Dawley (SD) rats (180 - 220 g) and male Kunming mice (25 - 30 g) were purchased from Laboratory Animal Centre of Sichuan University (Chengdu, China). Male New Zealand rabbits were purchased from Sichuan Special Committee of Farm Animals (Chengdu, China). Beagle dogs were provided by Dossy Biological Technology Co., Ltd (Chengdu, China). All animal protocols and experiments were approved by the Ethics Committee of Sichuan University and conducted in accordance with institutional guidelines.

\subsection{Preparation and characterization of OCT-PPSG}

OCT which was accurately weighed at $100 \mathrm{mg}$ was then dissolved in $0.75 \mathrm{~g} 85 \%$ ethanol $(v / v)$, followed by addition of $5 \mathrm{mg}$ glycine, $3.5 \mathrm{~g}$ E80 and $0.75 \mathrm{~g}$ injection-grade soybean oil. The mixture was stirred at room temperature for $30-60 \mathrm{~min}$ and filtered by $0.22 \mu \mathrm{m}$ microfiltration membrane before use. The viscosities of OCT-PPSG at varying temperatures were determined using a digital viscometer (DV-C, Brookfield Engineering Laboratories, Inc., USA).

\subsection{In vitro release studies}

OCT-PPSG which was accurately weighed at $200 \mathrm{mg}$ or $1 \mathrm{~mL}$ of OCT-soln (containing 1 $\mathrm{mg} / \mathrm{mL}$ OCT) were put into the dialysis bags (with molecular weight cut-off of 8-14 kD). The sample loaded dialysis bags were soaked in $4 \mathrm{~mL}$ of release medium $(0.01 \mathrm{M} \mathrm{pH7.4} \mathrm{PBS}$ containing varying percentages of ethanol), followed by shaking in a horizontal shaker (THZ-D, PeiYing, China) at $100 \mathrm{rpm}$ and $37^{\circ} \mathrm{C}$. At predetermined time intervals, the medium outside the dialysis bag was collected and replaced with $4 \mathrm{~mL}$ fresh medium. The collected medium was diluted to $5 \mathrm{~mL}$ by fresh medium and OCT concentration was determined by 
HPLC (Agilent 1260 infinity, USA).

\subsection{Determination of OCT}

Plasma samples were pretreated by protein precipitation method. Methanol was added at 4 times the volume of the plasma, shaken well and centrifuged at 12,000 g for $10 \mathrm{~min}$. The supernatant was filtered by $0.22-\mu \mathrm{m}$ microfiltration membrane for analysis. Prepared plasma samples were analyzed by HPLC tandem triple-quadrupole mass spectrometry (LC-MS/MS, Agilent Technologies-6410B, Santa Clara, CA, USA) with a diamonsil column $(50 \times 4.6 \mathrm{~mm}$, ODS, $1.8 \mu \mathrm{m}$ ). Mobile phases consisted of acetonitrile and $0.1 \%$ formic acid aqueous solution $(22: 78, v / v)$. The flow rate was $0.4 \mathrm{~mL} / \mathrm{min}$. The column was maintained at $30^{\circ} \mathrm{C}$ and $3 \mu \mathrm{L}$ of each sample was injected into the system for analysis. The mass spectrometer was operated by an electrospray source configured to positive ion mode, and the quantification analysis was performed by multiple reaction monitoring (MRM). The precursor ion of the analyte was 510 . The fragmentor was $120 \mathrm{eV}$. The instrumental parameters were listed as follows: gas temperature was $350{ }^{\circ} \mathrm{C}$, nebulizer was $30 \mathrm{psi}$, gas flow was $10 \mathrm{~mL} / \mathrm{min}$, and capillary was $4000 \mathrm{~V}[32,33]$.

\subsection{In vivo pharmacokinetic study}

\subsubsection{Pharmacokinetic study in rats}

Twelve male SD rats were divided into two groups, and housed in standard cages at $25^{\circ} \mathrm{C}$, with free access to food and water for a week prior to the experiment. Rats were subcutaneously injected with octreotide solution (OCT-soln) or OCT-PPSG at an equivalent single dose of $20 \mathrm{mg} / \mathrm{kg}$. The dose was determined based on the clinical dose of OCT-soln (Sandostatin) in human [34]. Rats were fasted for $12 \mathrm{~h}$ before dosing and food was returned approximately $2 \mathrm{~h}$ post dosing. Blood samples were collected at predetermined time points using heparinized Eppendorf tubes. Immediately after collection, the blood samples were placed on ice until centrifuged at 3,000 g for 10 min within $1 \mathrm{~h}$. The plasma was collected and stored at $-20^{\circ} \mathrm{C}$ until analysis.

\subsubsection{Pharmacokinetic study in rabbits}

Twelve male New Zealand rabbits were randomly divided into two groups. During the experiments, the rabbits were placed in the restraining boxes, and their heads were allowed to move freely. OCT-soln or OCT-PPSG formulations were administered in rabbits via subcutaneous injection. OCT-soln was administered as a single dose at an equivalent dose of $0.025 \mathrm{mg} / \mathrm{kg}$ [35]. Seven hundred microliter of OCT-PPSG $(4 \mathrm{mg} / \mathrm{mL})$ was administered as a single dose. After administration, these rabbits were put back into cages and allowed access to food $2 \mathrm{~h}$ post dosing. Blood samples were collected at predetermined time points via rabbit auricular veins and placed on ice before immediate centrifugation. Heparin in saline was used to flush the cannula before each collection of blood [36]. Blood samples were immediately centrifuged at 3,000 g for $10 \mathrm{~min}$ and the plasma was then collected and stored at $-20^{\circ} \mathrm{C}$ until analysis.

\subsubsection{Pharmacokinetic study in Beagle dogs}

Beagle dogs weighing 9 - $11 \mathrm{~kg}$ were housed in an environmentally controlled room with 
free access to food and water prior to experiment. Nine male and nine female Beagle dogs were divided into three groups with three male and three female dogs in each group. Beagle dogs were fasted for $12 \mathrm{~h}$ before dosing and water was available ad libitum. Food was returned approximately $4 \mathrm{~h}$ post dosing. Three formulations were administered as follows: OCT-soln at a single dose of $0.022 \mathrm{mg} / \mathrm{kg}(0.3 \mathrm{mg} / \mathrm{mL}$, s.c.); OCT-PPSG at a single dose of $1.4 \mathrm{mg} / \mathrm{kg}\left(20 \mathrm{mg} / \mathrm{mL}\right.$, s.c.) and Sandostatin ${ }^{\circledR} \mathrm{LAR}$ at an equivalent dose of $1.4 \mathrm{mg} / \mathrm{kg}$ (20 $\mathrm{mg} / \mathrm{mL}$, i.m.) [37]. About $2-3 \mathrm{~mL}$ of Blood samples were collected at $0.08,0.25,0.5,0.75,1$, 2, $3 \mathrm{~h}$ post OCT-soln administration and at $0.08,0.25,0.5,1,2,4,6,8,12,24,48,72,96,120$, 144, 168, 192, 216, 240, 288, 336, 384, 432, 480, 528, 624, 720 and 814 h post OCT-PPSG and LAR administration. Heparinized tubes were used to collect blood samples. Blood samples were immediately centrifuged at $3000 \mathrm{~g}$ for $10 \mathrm{~min}$ to collect plasma which was stored at $-20^{\circ} \mathrm{C}$ until analysis.

\subsection{Construction of in vitro-in vivo correlation (IVIVC)}

The Wagner-Nelson method was used to de-convolute the in vivo pharmacokinetic data assuming a one-compartment distribution for OCT-PPSG to determine the percent of drug absorption (absorption\%). The equation of the Wagner-Nelson relationship is displayed as Eq.(1):

$$
\text { Absorption } \%=\frac{C_{t}+K_{e} \cdot A U C_{0 \rightarrow t}}{K_{e} \cdot A U C_{0 \rightarrow t}} \times 100 \%
$$

where $C_{t}$ represents the concentration of octreotide in plasma at a certain time (t), $K_{e}$ is the elimination rate constant, $A U C_{0 \rightarrow t}$ is the area under the plasma concentration curve from time 0 to t, and $A U C_{0 \rightarrow \infty}$ is the area under the plasma concentration curve from time 0 to infinity [38]. The pharmacokinetic parameters of OCT-PPSG were obtained using DAS 3.2.5 software (China).

The in vitro release\% was calculated by Eq.(2):

$$
\text { Release } \%=\frac{C_{t}}{C_{0}} \times 100 \%
$$

where $C_{t}$, determined in release medium at a certain time $(\mathrm{t})$, is the concentration of octreotide which released from OCT-PPSG; $C_{0}$ is the estimated total concentration of octreotide based on the formulation of OCT-PPSG.

The in vitro release $\%$ and in vivo absorption $\%$ of rats, rabbits and beagles were used to construct IVIVC models for OCT-PPSG. The IVIVC was generated by plotting the in vitro release $\%$ on the $\mathrm{X}$-axis and the corresponding in vivo absorption\% on the Y-axis at same time points using Origin ${ }^{\circledR} 7.0$ software (USA). The regression coefficients were generated to select the best model by linear and polynomial regressions.

\subsection{In vivo pharmacodynamic study}

\subsubsection{Hepatocellular carcinoma xenografts in mice}

One mouse was randomly selected to receive intraperitoneal (i.p) injection of H22 hepatoma cells (Chinese Academy of Science Cell Bank for Type Culture Collection, Shanghai, China). One week later, the mouse with H22 hepatoma cells was sacrificed and the ascites were collected as H22 hepatoma cell suspension for use. Male Kunming mice (25 - 30 g) were subcutaneously injected with $\mathrm{H} 22$ hepatoma cell suspension $\left(2.0 \times 10^{6}\right.$ cells in $200 \mu \mathrm{L}$ 
of normal saline) to establish the tumor xenograft mouse model as previously described [39].

Thirty mice with HCC xenografts were randomly divided into three groups: (A) OCT-soln group, (B) OCT-PPSG group, and (C) control group. OCT-soln group received i.p injection of $100 \mu \mathrm{g} / \mathrm{kg}$ OCT-soln once a day and totally for consecutive 14 days. OCT-PPSG group received a single subcutaneous injection of $1.4 \mathrm{mg} / \mathrm{kg}$ OCT-PPSG, and the injection volume was about $0.2 \mathrm{~mL}$. Control group received i.p. injection of saline once a day for consecutive 14 days. Treatment started on the next day after injection of H22 hepatoma cell suspension and maintained for 14 days. Tumor growth was monitored by periodic caliper measurements on day 7 and day 14 post seeding. Tumor volumes (V) were calculated based on the length and width of tumor by Eq.(3) [40]:

$$
V=\left[\text { length } \times(\text { width })^{2}\right] / 2
$$

Inhibitory rate\% was calculated by Eq.(4):

$$
\text { Inhibitory rate } \%=100 \% \times\left(\mathrm{V}_{\text {control }}-\mathrm{V}_{\text {experimental group }}\right) / \mathrm{V}_{\text {control }}
$$

Survival experiment Thirty male Kunming mice with hepatocellular carcinoma xenografts were randomly divided into three groups: saline (NC, 0.9\% NaCl), OCT-PPSG, and OCT-soln. Saline group received i.p. injection of NC once a day. OCT-PPSG group received subcutaneous injection of OCT-PPSG at a dose of $1.4 \mathrm{mg} / \mathrm{kg}$. OCT-soln group received i.p injection of $100 \mu \mathrm{g} / \mathrm{kg}$ OCT-soln once a day. Treatment started on the day 7 after the injection of $\mathrm{H} 22$ hepatoma cell suspension and continued for 14 consecutive days. From the day 22, the survival condition of each group was observed and recorded every day, and the experiment was terminated when mice in the same group all deceased. The survival rate of each group at different time points and the median survival time of each group were calculated to graph the survival curve.

Immunohistological analysis Animals in the antitumor effect study were weighed and sacrificed at the day 14 after the initial treatment. Histological analysis was performed on collected tumors, and a portion of the specimen was fixed in $4 \%$ paraformaldehyde and embedded in paraffin wax, then sectioned at $4 \mu \mathrm{m}$ thickness and stained with hematoxylin and eosin (H\&E). Apoptotic cells were stained with terminal deoxynucleotidyl transferase dUTP nick end labeling (TUNEL; Roche Diagnostics GmbH, Germany) per manufacturer's protocol [41, 42]. Ki-67 [43, 44] and CD31 [45-47] proteins were also stained to gain insights into the proliferation of tumor cells and tumor-related angiogenesis. Light microscopy was used to evaluate pathological condition of tumors. The percentage of positively stained cells $(<25 \%$, $25 \%-50 \%, 50 \%-75 \%$, > 75\% were defined as “-” “+” “++” “+++”, respectively) or number of blood vessels in each visual field were calculated for quantitative comparisons.

\subsubsection{Effects on hepatocellular carcinoma induced by DEN and NMOR in rats}

Establishment of hepatocellular carcinoma in Sprague-Dawley rats Male SD rats were randomly divided into the following groups: normal, saline, OCT-soln, OCT-PPSG. Animals in normal group were fed with standard diet and pure drinking water for the whole period of experiment. Animals in other three groups received i.p. injection of $100 \mathrm{mg} / \mathrm{kg}$ diethylnirtrosamine (DEN, FBMVM-SA, TCI, Tokyo chemical industry, Japan) after adapted to the housing environment for one week. Animals were given drinking water containing 100 mg/L of N-nitrosomorpholine (NMOR, 5674K-AC, TCI, Tokyo chemical industry, Japan) for 8 weeks and the carcinogen solution was renewed every other day. From week 9 till the end 
of the experiment, animals were given pure drinking water only.

Pharmacodynamic study Saline group received subcutaneously injection of $1 \mathrm{~mL} / \mathrm{kg}$ NC only. OCT-soln treatment group was given subcutaneous injection of $0.2 \mathrm{mg} / \mathrm{kg}$ body weight of OCT-soln every other day from the beginning of the experiment to the end of experiment in the week 16 [48]. OCT-PPSG group received subcutaneous injection of $6 \mathrm{mg} / \mathrm{kg}$ OCT-PPSG, the injection volume of which was about $0.7 \mathrm{~mL}$, once a month from the beginning of the experiment to the end of experiment in the week 16. Normal group was subjected to no disposal.

In the week 16, all rats were sacrificed by femoral artery bleeding. The blood samples were collected from the experimental animals and liver tissue was removed and washed in ice-cold NC. The vascular endothelial growth factor (VEGF) was assayed in the serum using an enzyme-linked immunosorbent assay kit (Cloud-clone Corp L150310263, Houston, USA) by Varioskan Flash (Thermo Scientific, Finland) within three days. Numbers of tumor nodules per nodule-bearing liver were evaluated, and the body weight and liver weight were obtained at the end of experiment. Average liver weight index was calculated by Eq.(5):

Average liver weight index $\%=100 \% \times$ liver weight $/$ body weight

Histological evaluation was performed on a middle lobe of the liver and sections of $2-3$ $\mathrm{mm}$ thickness were fixed in fixative (glacial acetic acid : methanol : $70 \%$ ethanol $=1: 1: 18$ ) for $48 \mathrm{~h}$ and embedded in paraffin wax, sectioned at $4 \mu \mathrm{m}$ thickness and stained with hematoxylin-eosin. Light microscopy was used to evaluate pathological changes of liver.

Immunohistological analysis Tissue sections of $4 \mu \mathrm{m}$ thickness were dewaxed in xylene and rehydrated through graduated alcohol. Sections were subjected to heat-mediated antigen retrieval. Then sections were incubated overnight with primary antibodies at $4{ }^{\circ} \mathrm{C}$ (polyclonal rabbit anti-mouse SSTR2 (Lot 4926, No.37257, SAB, U.S.A; 1:100 dilution) and polyclonal rabbit anti-mouse SSTR5 (Lot 4926, No.37260, SAB, U.S.A; 1:100 dilution)). The secondary antibody for incubation was biotinylated horse anti-rabbit IgG (Vector Laboratories; 1:500 dilution). Detection was facilitated using the streptavidin-ABC (avidin/biotinylated enzyme complex)/horse radish peroxidase (HRP) system with NovaRED ${ }^{\mathrm{TM}}$ as the substrate (Vector Laboratories). Counterstaining was conducted with hematoxylin. The levels of SSTR2 and SSTR5 were determined by computerized morphometry of approximately 30 interstitial rectangular fields per section at $400 \times$ magnification.

\subsection{Statistical analysis and pharmacokinetic parameters}

All data were presented as mean \pm standard deviation (SD). The statistical analysis between two groups was performed using Student's $t$-test. Differences were considered statistically significant at $p<0.05$. The pharmacokinetic parameters were calculated by DAS 3.2.5 software (Chinese Pharmacological Association, Anhui, China). Comparisons among multiple groups were performed by one-way ANOVA followed by Tukey as the post hoc test with $p<0.05$ as the threshold of significance.

\section{Results}

\subsection{In vitro characterization}

The obtained OCT-PPSG pre-gel solution displayed in the appearance of a homogeneous, clear and bright orange solution with good fluidity (Fig. 1A and 1C). Using a dialysis setup, 
OCT-PPSG pre-gel solution achieved rapid phase-separation and transition into a gel-like material with a loss of fluidity (Fig. 1B and 1D). The viscosity of OCT-PPSG pre-gel solutions were slightly higher than that of blank PPSG at the same temperature (Fig. 1E). For both blank PPSG and OCT-PPSG pre-gel solutions, a decrease in viscosity was observed with increasing temperature.
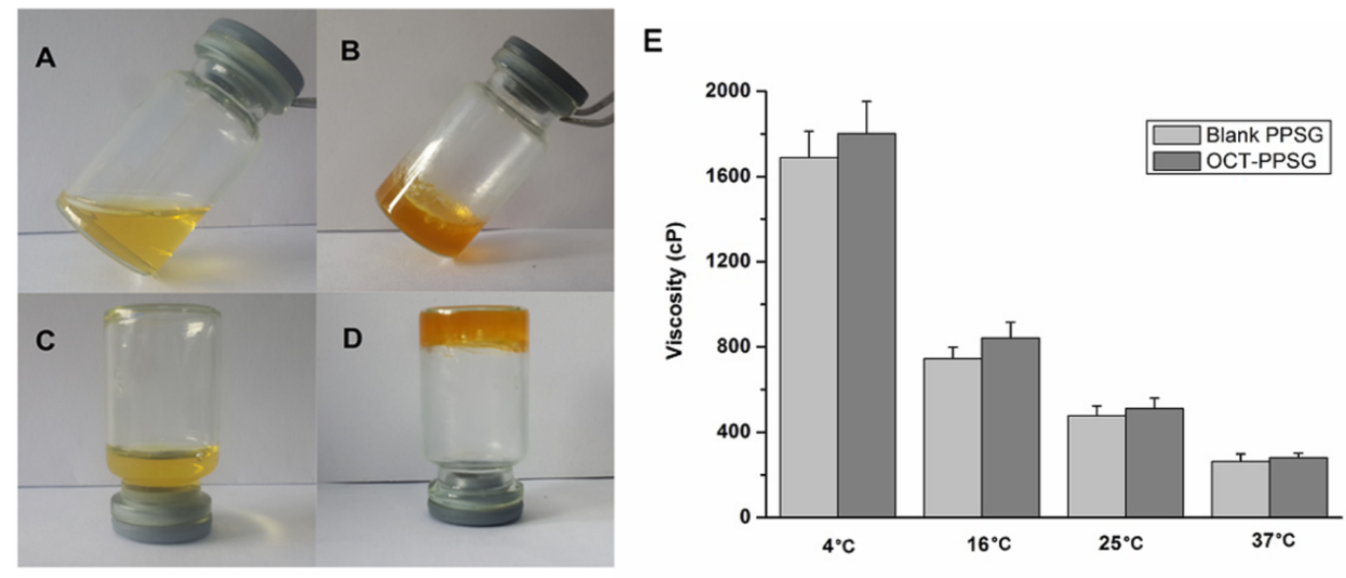

Fig. 1

\subsection{In vitro release}

Phosphate buffer saline (PBS, $0.01 \mathrm{M}, \mathrm{pH}$ 7.4) was used as release medium. Preliminary studies showed very low cumulative release of therapeutic compounds from the PPSG formulation. To mimic the degradation of PPSG gel material, release media with varying percentages of ethanol were used. The cumulative release of octreotide increased gradually with the increase of ethanol content in the release media ranging from 0 to $50 \%(v / v)$ (Fig. 2). OCT displayed the highest cumulative release at all given time points with $50 \%$ ethanol in the release medium, and a moderate cumulative release with $20 \%(\mathrm{v} / \mathrm{v})$ ethanol in the release medium (Fig. 2A). An ethanol-dependent cumulative release of OCT was observed by the day 10 (Fig. 2B). Results showed that the cumulative release percentages of OCT were of significant differences among release media with varying percentages of ethanol $(p<0.05)$. The cumulative release of OCT-soln was around 85\% within $6 \mathrm{~h}$ and above $90 \%$ at $12 \mathrm{~h}$ in PBS (Fig. 2C). In contrast, the release of OCT-PPSG followed a sustained and controlled manner, and the cumulative release of OCT from OCT-PPSG within $72 \mathrm{~h}$ was about $10 \%$ in PBS-only medium. OCT-soln displayed a similar release pattern as that in PBS when in the medium containing 20\% ethanol $(\mathrm{v} / \mathrm{v})$. But the release of OCT from OCT-PPSG was much faster (47\% cumulative release within $72 \mathrm{~h}$ ) than that in PBS, likely due to the higher solubility of phospholipids in 20\% ethanol (Fig. 2C and 2D). 

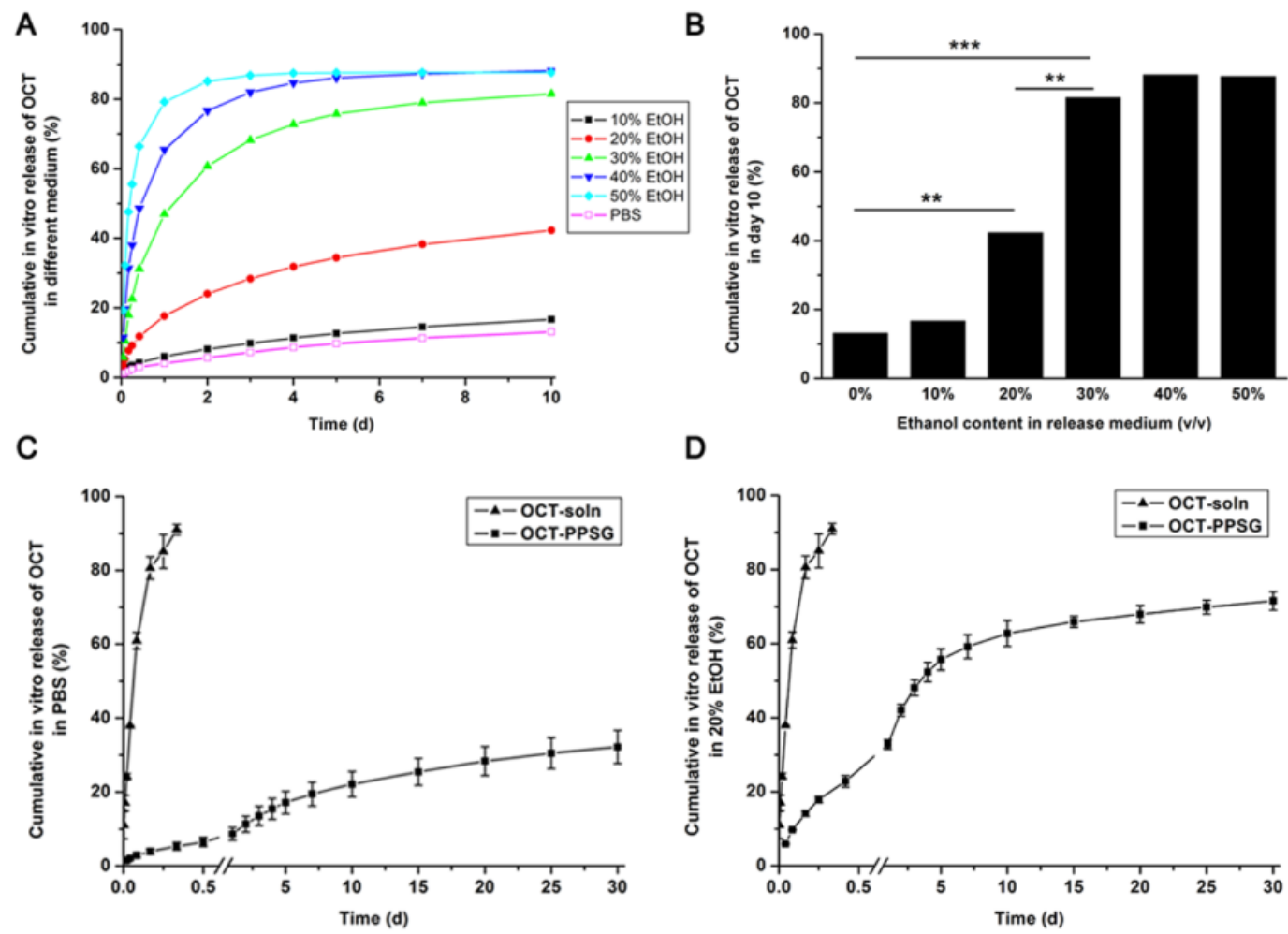

Fig. 2

\subsection{In vivo pharmacokinetic study}

\subsubsection{Pharmacokinetic study in rats}

Octreotide concentrations were determined at given time points, and concentration-time profiles were obtained (Fig. 3A). OCT-soln group displayed rapid accumulation in $\mathrm{C}_{\max }$ in 30 min and rapid elimination, the $\mathrm{MRT}_{0_{-\infty}}$ of which was $6.95 \mathrm{~h}$. In comparison, OCT-PPSG showed a relative slow and steady decrease of octreotide concentration over time, the $\mathrm{MRT}_{0-\infty}$ of which was $341.4 \mathrm{~h}$. Besides, OCT-PPSG showed a greater exposure with an $\mathrm{AUC}_{0-\infty}$ value of $3511.24 \mu \mathrm{g} \cdot \mathrm{h} / \mathrm{L}$ than OCT-soln $(208.66 \mu \mathrm{g} \cdot \mathrm{h} / \mathrm{L})$. The results were consistent with the previous findings [21], and OCT-PPSG was observed to achieve sustained octreotide release for up to $30 \mathrm{~d}$ in rats.

Table 1. OCT plasma pharmacokinetic parameters after single administration of OCT-soln and OCT-PPSG in rats.

\begin{tabular}{lcc}
\hline & OCT-soln & OCT-PPSG \\
\hline $\mathrm{C}_{\max }(\mu \mathrm{g} / \mathrm{L})$ & $32.2 \pm 1.86$ & $26.7 \pm 6.5$ \\
$\mathrm{t}_{\max }(\mathrm{h})$ & 0.5 & 1.0 \\
$\mathrm{t}_{1 / 2 \mathrm{Z}}(\mathrm{h})$ & 12.6 & 296.2 \\
$\mathrm{AUC}_{(0-\infty)}(\mu \mathrm{g} \cdot \mathrm{h} / \mathrm{L})$ & 208.66 & 3511.24 \\
$\operatorname{MRT}_{(0-\infty)}(\mathrm{h})$ & 6.95 & 341.4 \\
\hline
\end{tabular}

\subsubsection{Pharmacokinetic study in rabbits}

After a single subcutaneous injection in rabbits, the pharmacokinetic profiles of OCT-soln 
$(0.025 \mathrm{mg} / \mathrm{kg})$ and OCT-PPSG (1.4 mg/kg) were obtained (Fig.3B). A sharp initial increase in plasma concentration of OCT was observed in OCT-soln group, reaching $\mathrm{C}_{\max }$ of $15.2 \mu \mathrm{g} / \mathrm{L}$ at $0.5 \mathrm{~h}$ followed by a relatively rapid decrease in plasma concentration in the elimination phase. In contrast, OCT-PPSG displayed significantly longer MRT $_{0-\infty}(261.8 \mathrm{~h})$ and half-life (164.35 h). Meanwhile, OCT-PPSG showed a much higher $\mathrm{AUC}_{0-\infty}$ value of $363.1 \mu \mathrm{g} \cdot \mathrm{h} / \mathrm{L}$ than OCT-soln $(18.9 \mu \mathrm{g} \cdot \mathrm{h} / \mathrm{L})$.

Table 2. OCT plasma pharmacokinetic parameters after single administration of OCT-soln and OCT-PPSG in rabbits.

\begin{tabular}{lcc}
\hline & OCT-soln & OCT-PPSG \\
\hline $\mathrm{C}_{\max }(\mu \mathrm{g} / \mathrm{L})$ & $15.2 \pm 0.93$ & $8.16 \pm 4.22$ \\
$\mathrm{t}_{\max }(\mathrm{h})$ & 0.5 & 0.8 \\
$\mathrm{t}_{1 / 2 Z}(\mathrm{~h})$ & 0.567 & 164.35 \\
$\mathrm{AUC}_{(0-\infty)}(\mu \mathrm{g} \cdot \mathrm{h} / \mathrm{L})$ & 18.9 & 363.1 \\
$\mathrm{MRT}_{(0-\infty)}(\mathrm{h})$ & 0.936 & 261.8 \\
\hline
\end{tabular}

\subsubsection{Pharmacokinetic study in Beagles}

The pharmacokinetic profiles of OCT-soln, OCT-PPSG and Sandostatin LAR ${ }^{\circledR}$ in Beagle dogs were obtained following a single subcutaneous injection (Fig.3C). Plasma concentrations of OCT were monitored for $3 \mathrm{~h}, 720 \mathrm{~h}$, and $720 \mathrm{~h}$ for OCT-soln, OCT-PPSG and Sandostatin $\mathrm{LAR}^{\circledR}$, respectively. The moderate release at early stage in Sandostatin LAR ${ }^{\circledR}$ is evidenced by $\mathrm{C}_{\max }$ and $t_{\max }$ values of $13.98 \pm 5.88 \mu \mathrm{g} / \mathrm{L}$ and $0.5 \pm 0.27 \mathrm{~h}$. In contrast, the concentration-time profile of OCT-PPSG remained relatively stable as expected with a lower $\mathrm{C}_{\max }$ of $7.37 \pm 2.56 \mu \mathrm{g} / \mathrm{L}$ and a longer $\mathrm{t}_{\max }$ of $1.75 \pm 0.61 \mathrm{~h}$. Meanwhile, the plasma concentration of OCT in OCT-PPSG remained much higher than that after administration of Sandostatin $\mathrm{LAR}^{\circledR}$ from $1 \mathrm{~h}$ to $168 \mathrm{~h}$. Afterwards, a gradual and steady decrease of OCT concentration was observed in OCT-PPSG while fluctuations of OCT concentration were observed in Sandostatin $\mathrm{LAR}^{\circledR}$ from $192 \mathrm{~h}$ to 720 h. Sandostatin $\mathrm{LAR}^{\circledR}$ displayed an $\mathrm{AUC}_{0-\infty}$ of $480.7 \pm 342.2 \mu \mathrm{g} \cdot \mathrm{h} / \mathrm{L}$, while OCT-PPSG showed a comparable $\mathrm{AUC}_{0-\infty}$ of $573.3 \pm 230.1$ $\mu \mathrm{g} \cdot \mathrm{h} / \mathrm{L}(p>0.05)$.

Table 3. OCT plasma pharmacokinetic parameters after single administration of OCT-soln, OCT-PPSG and Sandostatin LAR ${ }^{\circledR}$ in beagles.

\begin{tabular}{lccc}
\hline & OCT-soln & OCT-PPSG & Sandostatin LAR \\
\hline $\mathrm{C}_{\max }(\mu \mathrm{g} / \mathrm{L})$ & $21.83 \pm 3.86$ & $7.37 \pm 2.56$ & $13.98 \pm 5.88$ \\
$\mathrm{t}_{\max }(\mathrm{h})$ & $0.35 \pm 0.13$ & $1.75 \pm 0.61$ & $0.5 \pm 0.27$ \\
$\mathrm{t}_{1 / 2 Z}(\mathrm{~h})$ & $0.66 \pm 0.29$ & $367.5 \pm 223.8$ & $225.2 \pm 119.8$ \\
$\mathrm{AUC}_{(0-\infty)}(\mu \mathrm{g} \cdot \mathrm{h} / \mathrm{L})$ & $29.72 \pm 4.13$ & $573.3 \pm 230.1$ & $480.7 \pm 342.2$ \\
$\mathrm{MRT}_{(0-\infty)}(\mathrm{h})$ & $1.15 \pm 0.42$ & $279.14 \pm 141.2$ & $446.8 \pm 97.5$ \\
\hline
\end{tabular}


A
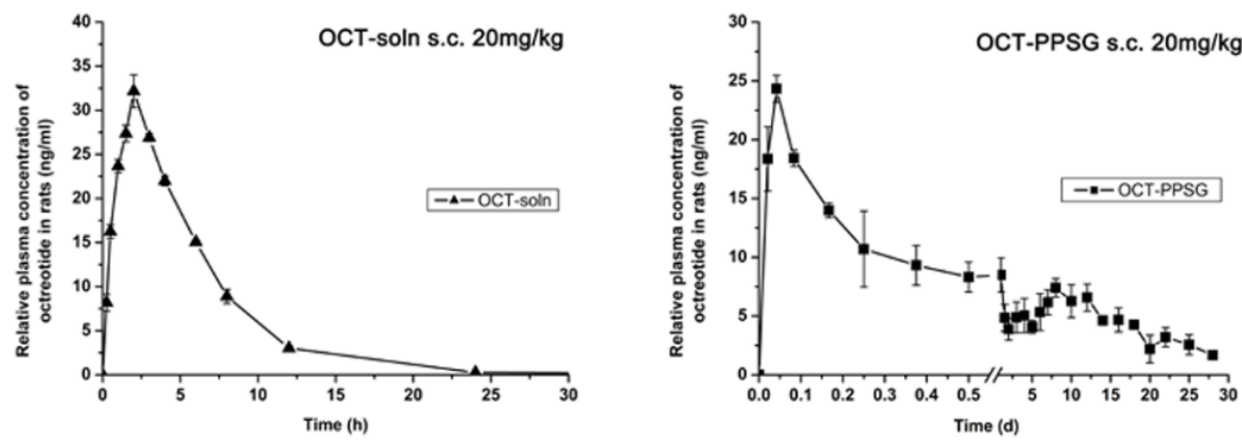

B
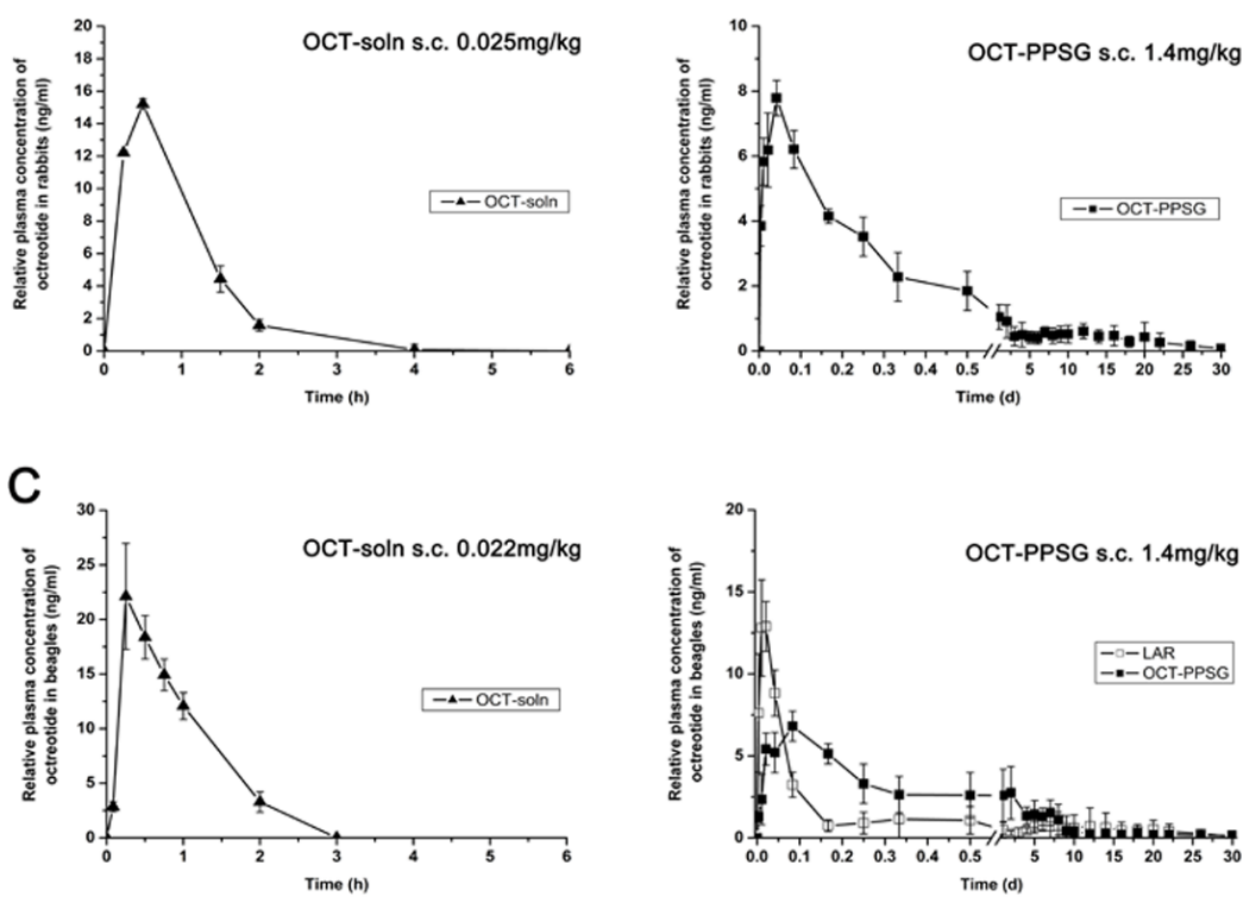

Fig. 3

\subsection{IVIVC model}

Based upon the in vitro release $\%$ and in vivo absorption\% data obtained from different animals, three IVIVC models were constructed. A second order polynomial correlation was found between the in vitro release\% (X) and in vivo absorption\% (Y) with the correlation coefficient $\left(R^{2}\right)$ values ranging from 0.8534 to 0.9901 (Fig. 4 and Table 4). The best correlation was obtained between the release data with $20 \%$ ethanol in the release medium and pharmacokinetic results in rabbits $\left(R^{2}=0.9901\right)$, followed by beagles and rats. Moreover, the increase of ethanol content in the release medium from $10 \%$ to $50 \%(\mathrm{v} / \mathrm{v})$ appeared to result in a decrease of correlation (Table 5), which was likely due to the accelerated degradation of phospholipids at high percentages of ethanol. 

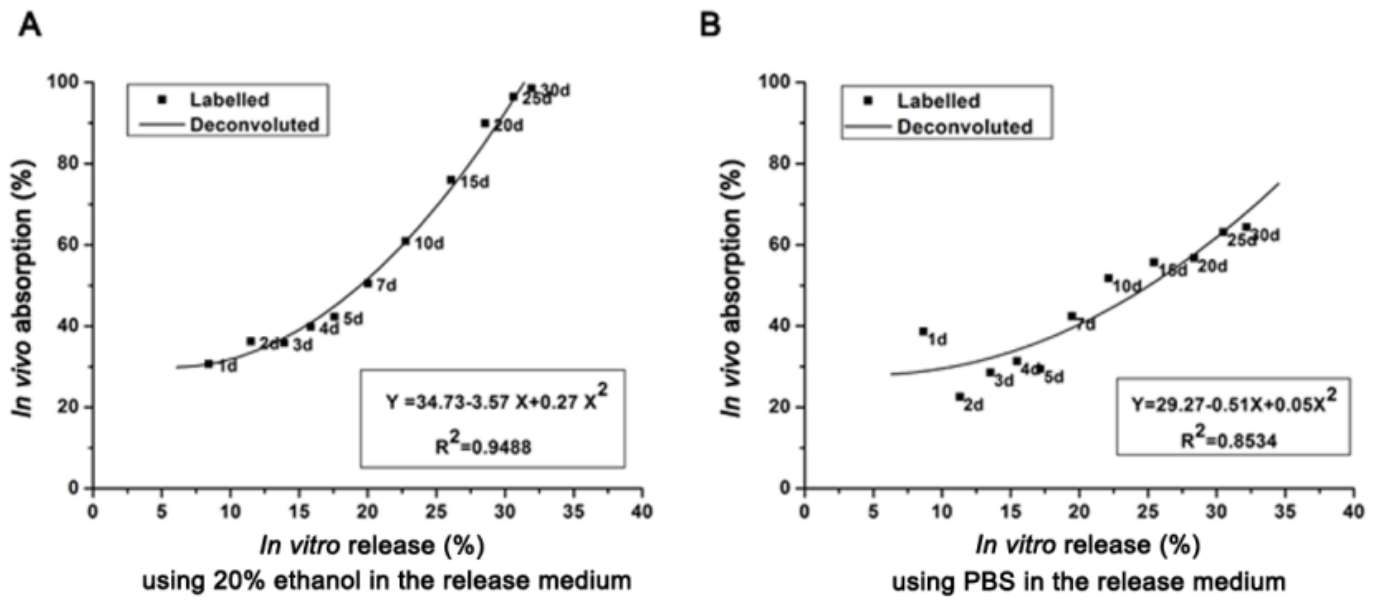

Fig. 4

Table 4. IVIVC models constructed using in vivo absorption\% and in vitro release\% of OCT in PBS and 20\% EtOH for three animal species.

\begin{tabular}{lccc}
\hline Animal & Release medium & IVIVC model & $R^{2}$ \\
\hline Rats & & $\mathrm{Y}=0.05 \mathrm{X}^{2}-0.51 \mathrm{X}+29.27$ & 0.8534 \\
Rabbits & PBS & $\mathrm{Y}=0.102 \mathrm{X}^{2}-1.12 \mathrm{X}+34.48$ & 0.9856 \\
Beagles & & $\mathrm{Y}=-0.01 \mathrm{X}^{2}+0.95 \mathrm{X}+7.97$ & 0.9394 \\
Rats & & $\mathrm{Y}=0.08 \mathrm{X}^{2}-1.85 \mathrm{X}+41.75$ & 0.8614 \\
Rabbits & \multirow{2}{*}{ 20\% EtOH } & $\mathrm{Y}=0.12 \mathrm{X}^{2}-1.73 \mathrm{X}+38.72$ & 0.9901 \\
Beagles & & $\mathrm{Y}=-0.009 \mathrm{X}^{2}+0.8482 \mathrm{X}+8.74$ & 0.9405 \\
\hline
\end{tabular}

Percentage numbers indicate the amount of ethanol in PBS (v/v) for in vitro studies.

Table 5. IVIVC constructed using in vivo absorption\% and in vitro release\% of OCT in different release medium for three kinds of animals

\begin{tabular}{cccc}
\hline Animal & Release medium & IVIVC model & $R^{2}$ \\
\hline \multirow{2}{*}{ Rats } & $10 \% \mathrm{EtOH}$ & $\mathrm{Y}=0.27 \mathrm{X}^{2}-3.57 \mathrm{X}+34.73$ & 0.9488 \\
& $20 \% \mathrm{EtOH}$ & $\mathrm{Y}=0.07 \mathrm{X}^{2}-3.3 \mathrm{X}+61.15$ & 0.9479 \\
$30 \% \mathrm{EtOH}$ & $\mathrm{Y}=0.02 \mathrm{X}^{2}-3.08 \mathrm{X}+107.3$ & 0.9149 \\
& $40 \% \mathrm{EtOH}$ & $\mathrm{Y}=0.02 \mathrm{X}^{2}-2.89 \mathrm{X}+126.3$ & 0.9042 \\
$50 \% \mathrm{EtOH}$ & $\mathrm{Y}=0.015 \mathrm{X}^{2}-2.56 \mathrm{X}+141.86$ & 0.8837 \\
& & & 0.9832 \\
& $10 \% \mathrm{EtOH}$ & $\mathrm{Y}=0.22 \mathrm{X}^{2}-2.39 \mathrm{X}+38.32$ & 0.9745 \\
$20 \% \mathrm{EtOH}$ & $\mathrm{Y}=0.05 \mathrm{X}^{2}-2.05 \mathrm{X}+51.7$ & 0.9633 \\
$30 \% \mathrm{EtOH}$ & $\mathrm{Y}=0.03 \mathrm{X}^{2}-3.01 \mathrm{X}+103.07$ & 0.9268 \\
& $40 \% \mathrm{EtOH}$ & $\mathrm{Y}=0.02 \mathrm{X}^{2}-2.98 \mathrm{X}+123.4$ & 0.8253 \\
& $50 \% \mathrm{EtOH}$ & $\mathrm{Y}=0.02 \mathrm{X}^{2}-2.73 \mathrm{X}+139.6$ & \\
& & & 0.8925 \\
& $10 \% \mathrm{EtOH}$ & $\mathrm{Y}=0.02 \mathrm{X}^{2}+0.51 \mathrm{X}+11.11$ & 0.8865
\end{tabular}




$\begin{array}{lll}30 \% \mathrm{EtOH} & \mathrm{Y}=0.003 \mathrm{X}^{2}-0.12 \mathrm{X}+14.34 & 0.8527 \\ 40 \% \mathrm{EtOH} & \mathrm{Y}=0.006 \mathrm{X}^{2}-0.56 \mathrm{X}+26.88 & 0.8222 \\ 50 \% \mathrm{EtOH} & \mathrm{Y}=0.03 \mathrm{X}^{2}-4.32 \mathrm{X}+165.75 & 0.7734\end{array}$

Percentage numbers indicate the amount of ethanol in PBS $(v / v)$ for in vitro studies.

\subsection{In vivo pharmacodynamics study}

\subsubsection{Effects on hepatocellular carcinoma xenografts in mice}

The antitumor effects of various OCT formulations were evaluated in the mice H22 xenografts. The tumor volume in cubic millimeter of saline, OCT-soln and OCT-PPSG group of animals were obtained (Fig. 5B). Both OCT-treated groups showed a significant reduction in the tumor volume when compared with saline group $(p<0.05)$. OCT-PPSG showed greater antitumor effect than OCT-soln, which was evidenced by average tumor volume below 30 $\mathrm{mm}^{3}$ on the day 14. No obvious changes were observed between the control and OCT-treated group which is an indicative of nontoxic nature of OCT formulations (Fig.5C). The inhibitory rates for both OCT formulations and the inhibitory rates of both formulations were above $85 \%$ on the day 7, 10, 12 and 14 (Fig. 5D). The antitumor effect remained comparable by the day 12. However, OCT-PPSG showed greater inhibitory effect than OCT-soln on the day 14 which was likely attributed to the long acting effect of octreotide. Tumor-bearing mice in the saline group all died by day 50 , while $30 \%$ of OCT-PPSG group and $10 \%$ of OCT-soln group remained alive by day 50. The median survival times of saline group, OCT-soln group and OCT-PPSG group were 23.3, 23.3 and 27 days, respectively (Fig. 5E).

A

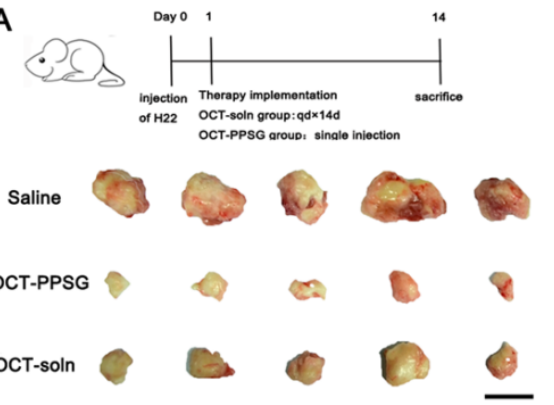

C

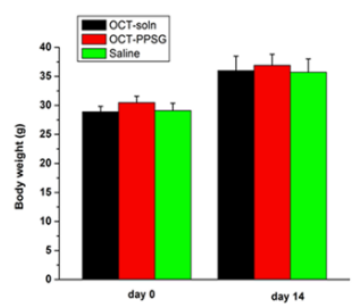

D

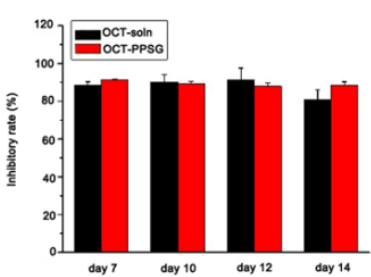

E
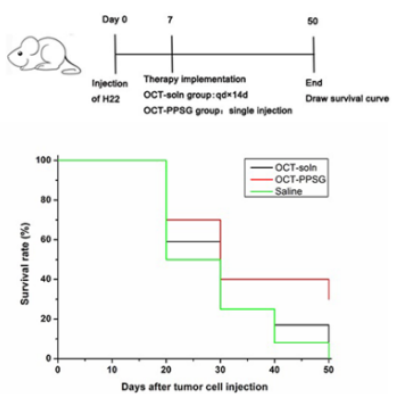

Fig. 5

According to the histological analysis, OCT-soln group displayed tumor necrosis and cell debris in the remaining tumor sections (Fig. 6A). In comparison, OCT-PPSG group showed 
more remarkable tumor necrosis and little cell debris. Unlike OCT treated groups, the tumor cells in the saline group displayed lamellar growth pattern with neoplastic avascular necrosis within tumor tissues. Cellular differentiation remained low which indicates high levels of malignancy and hyperchromatic cells were observed with large proportions of nucleoplasm. Significant nuclear division was not observed. Next, tumor sections were immunostained with anti CD31, Ki-67 and TUNEL antibodies to determine whether OCT treatment affects vascularization, cell proliferation and apoptosis in tumor xenografts (Fig. 6B). The level of CD31 was significantly higher in the saline group (49.2 \pm 31.6 strips per visual field) than that in the OCT-soln group ( $4.2 \pm 4$ strips per visual field) or the OCT-PPSG group ( $2.6 \pm 4.8$ strips per visual field). The level of Ki-67-positive cells in the OCT-PPSG group remained below $10 \%(-)$, which was remarkably lower than in the saline group $(\sim 50 \%,++)$ and the OCT-soln group $(\sim 30 \%,+)$. In the TUNEL experiment, the levels of positively stained cells in the OCT-soln, OCT-PPSG, and saline group were $80 \%(+++), 58 \%(++), 36 \%\left(^{+}\right)$, respectively, which strongly supported that OCT treatment led to tumor cell apoptosis.
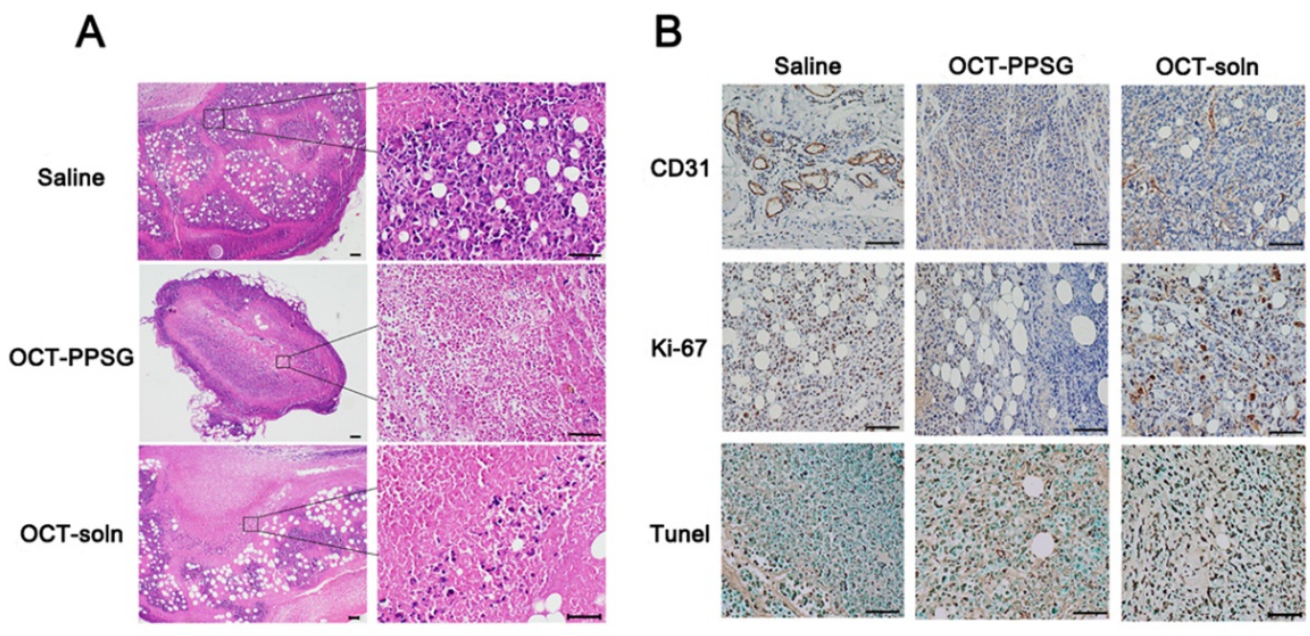

Fig. 6

\subsubsection{Effects on DEN- and NMOR- induced hepatocellular carcinoma in rats}

To further evaluate the antitumor efficacy of OCT-PPSG in vivo, a primary hepatocellular carcinoma model was established in SD rats. Affected by DEN and NMOR, rats started to lose hair and some displayed red-ring-like hair in necks after 8 weeks. For both OCT-soln group and OCT-PPSG group, the degree of hair change was less obvious compared to the saline group. After 12-16 weeks, all rats in the DEN and NMOR treated groups appeared to lose their appetite.

All animals were sacrificed at the end of experiment and their livers were excised and photographed. Macroscopic appearance of the liver of normal group animals showed normal morphology (Fig. 7A). Rats in the saline group showed heavy enlargement and several grayish white nodules and foci on the peripheral surface of the liver, while the OCT-soln and OCT-PPSG groups showed less remarkable morphological changes and less nodule formation. Many persistent but tiny nodules in DEN/NMOR + OCT-soln pretreated group of rats showed 
the antitumor effect of OCT-soln with repeated administration (Fig. 7A). Most of the foci and nodules disappeared in the liver from DEN/NMOR + OCT-PPSG pretreated group of rats showed the advanced antitumor effect of OCT-PPSG (Fig. 7A). The average number of tumor nodules and the average liver weight indexes were summarized in tumor-bearing animals (Table 6). When compared with the saline group, the OCT-treated groups showed a significant decrease $(p<0.05)$ in the number of nodules and the average liver weight indexes, which was in accordance with previous study [49].

Table 6. Related index observed in hepatocellular carcinoma rats induced by DEN and NMOR (mean \pm SD).

\begin{tabular}{lccc}
\hline & $\begin{array}{c}\text { Body weight } \\
(\mathrm{g})\end{array}$ & $\begin{array}{c}\text { Average liver weight index } \\
(\%)\end{array}$ & $\begin{array}{c}\text { Average number of } \\
\text { tumor nodules }\end{array}$ \\
\hline OCT-soln & $339.12 \pm 26.52$ & $2.95 \pm 0.15^{*}$ & $8.69 \pm 5.29 * *$ \\
OCT-PPSG & $338.76 \pm 20.70$ & $3.18 \pm 0.20^{*}$ & $7.92 \pm 6.15^{* *}$ \\
Saline & $330.16 \pm 20.36$ & $4.57 \pm 0.98$ & $16.56 \pm 7.11$ \\
\hline
\end{tabular}

Average liver weight index $(\%)=100 \% \times$ liver weight $/$ body weight.

Compared to the saline group, ${ }^{*}, p<0.05 ;{ }^{* *}, p<0.01$.

Liver injury of hepatocellular carcinoma can be classified into four levels: negative, transforming foci, cancerous nodules, and cancer [50]. Each sample was evaluated and the percentages of each level in different groups $(n=13)$ were calculated (Table 7)., the percentage of cancer in saline group (38\%) was much higher than that in OCT-soln group (8\%) and in OCT-PPSG group (0\%), while the percentage of negative in saline group (0\%) was significantly lower than in OCT-soln group (46\%) and in OCT-PPSG group (31\%).

Table 7. Percentages of four injured levels in OCT-soln, OCT-PPSG and saline groups $(n=13)$

\begin{tabular}{lcccc}
\hline & Negative & $\begin{array}{c}\text { Transforming } \\
\text { foci }\end{array}$ & $\begin{array}{c}\text { Cancerous } \\
\text { nodules }\end{array}$ & Cancer \\
\hline OCT-soln & $46 \%$ & $23 \%$ & $23 \%$ & $8 \%$ \\
OCT-PPSG & $31 \%$ & $31 \%$ & $38 \%$ & $0 \%$ \\
Saline & $0 \%$ & $8 \%$ & $54 \%$ & $38 \%$ \\
\hline
\end{tabular}

Percentage $(\%)=100 \% \times$ number of rats in this group classified into each injured level $/$ total number of rats in this group

Histological examination of liver sections from normal group animals revealed normal morphology and cells with granulated cytoplasm and small uniform nuclei (Fig.7A). The saline group revealed loss of normal morphology and tumor cell nucleus remained enlarged and anachromasis. Cellular pleomorphism was obvious and tumor giant cells were observed (Fig. 7A). OCT-treated group showed few neoplastically transformed cells and hepatocytes maintaining near normal structure (Fig.7A).

The levels of vascular endothelial growth factor (VEGF) in serum were further determined by ELISA. The level of serum VEGF was much lower in OCT-soln group ( $p<$ 0.05 ) than that in the saline group, while the level of VEGF in OCT-PPSG group remained the lowest (Fig. 7B). These two mentioned results appeared in good agreement with each other, 
which suggested that octreotide might inhibit hepatocarcinogenesis by inhibiting VEGF secretion.

Moreover, OCT-treatments resulted in significant inhibitory effect on the expression levels of SSTR2 and SSTR5 in primary HCC-bearing rats compared with the saline group (Fig.7C and Fig.7D). OCT-PPSG appeared to inhibit the expression of SSTR2 and SSTR5 to a greater extent than that of OCT-soln treated group.
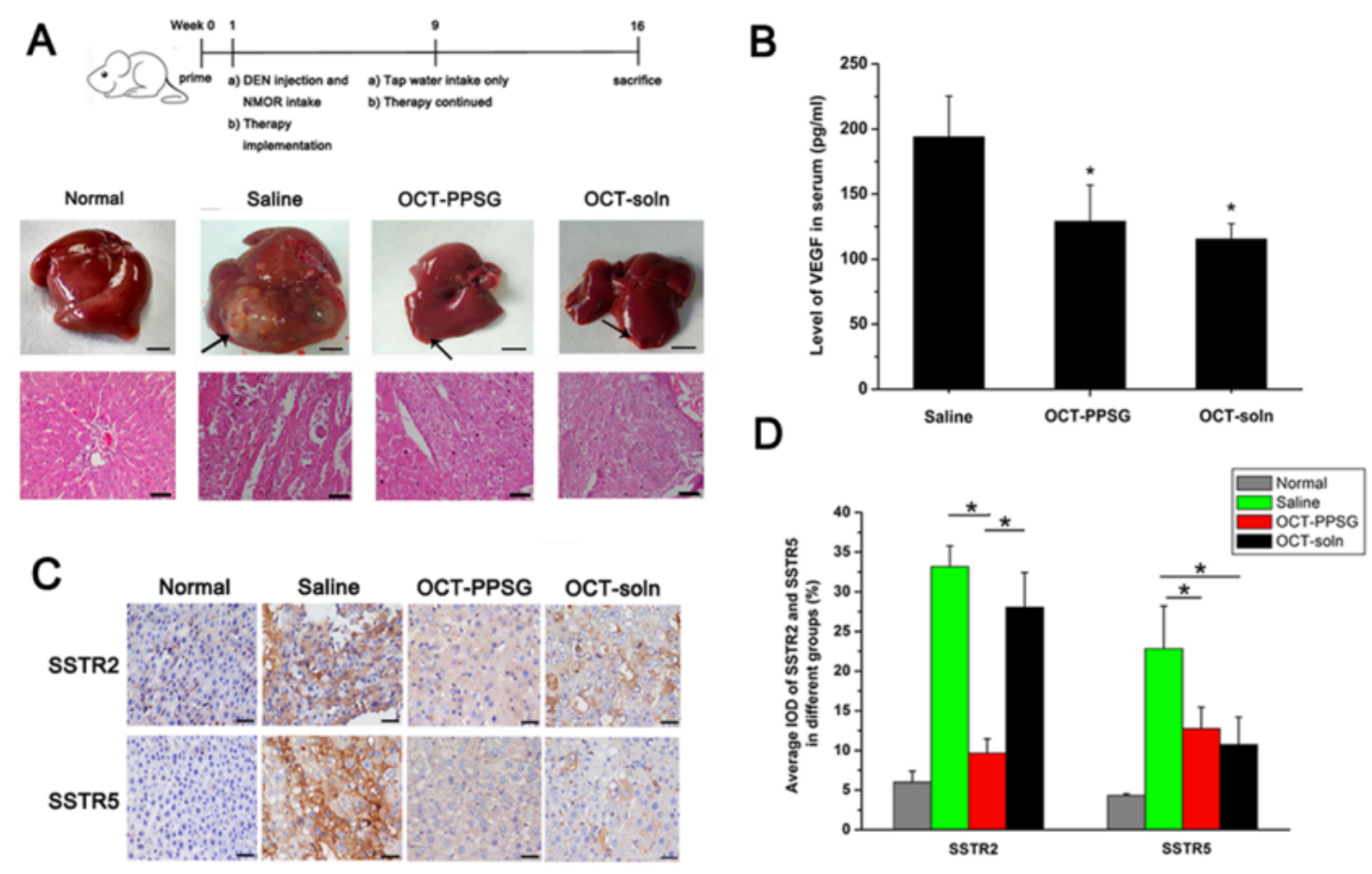

Fig. 7

\section{Discussion}

In the current study, we reported a readily injectable, biocompatible and efficient in situ forming gel platform material for the sustained delivery of OCT. Regarding the PPSG system, 85\% ethanol in PBS was used as the solvent instead of commonly used organic solvents in organogels (such as aliphatic ester, alkane, cyclane and amine) [51], thus rendering the materials less toxic and irritant. A previous study by Zhang $\mathrm{T}$ et al has evaluated the irritation of PPSG in rabbits, which showed the presence of ethanol may cause some but acceptable irritation in rabbits [21]. Since the percentage of ethanol in the current formulation has remained the same compared to our previous study, a similar level of irritation is expected for the current PPSG formulation.

Another major advantage of PPSG is its sustained release profile without remarkable initial burst effect. The initial burst release is a double-edged sword, which needs careful modulation for a controlled release formulation. Although burst release can provide an initial boost dose and thus rapidly reach therapeutic window, an exceedingly high burst release could result in adverse effects associated with high drug concentrations [21]. In the study, local viscosity, solvent diffusion and micro-structures of PPSG were possible impact factors contributing to the initial burst release. The less significant burst release might result from the 
limited amount of ethanol presented in the pre-gel solution $(\sim 12.4 \% \mathrm{w} / \mathrm{w})$, and therefore, the initial burst associated with ethanol diffusion would be limited. In addition, PPSG pre-gel solution containing high content of phospholipids was proven to possess low viscosity, which was highly desirable for injection through high gauge needles. After injection, PPSG rapidly underwent phase separation and transition into a gel like implant with dramatically increased viscosity.

In the current study, PPSG platform materials were applied to the long-term delivery of OCT, and the pharmacokinetic profiles of OCT were evaluated in animal models of three species, including SD rats, rabbits, and Beagle dogs. In all the three models, OCT-PPSG showed controlled and sustained in vivo release profiles for up to $30 \mathrm{~d}$. However, differences in pharmacokinetic parameters were observed. OCT-PPSG showed much lower initial burst release in Beagle dogs than that in rabbits with longer $t_{\max }$ and lower $C_{\max }$. Meanwhile, OCT displayed a shorter circulation time in rabbits than that in Beagle dogs with smaller $t_{1 / 2}$ and $\mathrm{AUC}_{0-\infty}$, which might be attributed to differences in digestive systems of the two species [35]. In addition, specie differences were observed in the IVIVC study. The correlation between in vitro release and in vivo absorption was evaluated by regression coefficient $\left(R^{2}\right)$ [38]. Rabbits showed the best correlation, which was possibly due to its animal size, injection dose, gene homology, routes of absorption, digestion, metabolism and elimination [52]. The differences observed in burst release of OCT-PPSG in vitro and in vivo were largely due to the setup of the in vitro release study, which might not be sufficient to mimic the in vivo conditions. Firstly, in the in vitro release study, the presence of ethanol in the release media was proven critical to the sustained release of solutes. Secondly, the PPSG did not undergo degradation in the in vitro release study, and the driving force for OCT release would be the concentration-gradient between the gel matrix and the release medium. OCT release was likely driven by solute diffusion. Regarding the in vivo release profiles, the initial burst was most likely due to the rapid diffusion of ethanol during gel formation. Also, no such sink condition could be maintained in the in vivo release conditions. To address these concerns, we sought to develop an in vitro release setup which could better correlate with the in vivo release condition [69]. Anyway, the lack of correlation between in vitro release setup and in vivo release conditions needed to be further acknowledged for pharmaceutical scientists when release studies were performed in vitro.

To evaluate the therapeutic efficacy of OCT-PPSG, we selected HCC as the disease model, which has been the fifth most common cancer in the world and the third most common cause of cancer-related death [53]. To reduce the morbidity and mortality of HCC, early diagnosis and development of novel systemic therapies for HCC prevention are of paramount importance. A primary HCC model was induced by DEN and NMOR in rats [54], which has been validated to have similar forming progression including hepatic toxic lesion, hepatic proliferation/cirrhosis, hepatic carcinogenesis and metastases as compared to human hepatocellular carcinomas [48, 54]. Primary hepatocellular carcinoma included two stages: early hepatocellular carcinoma and advanced hepatocellular carcinoma [55]. The tumors were often spherical in shape with clear boundary and tumor sections were homogeneous without bleeding and necrosis in early hepatocellular carcinoma [56, 57]. Advanced hepatocellular carcinoma showed three typical morphologies, i.e., giant, multinodular and diffuse, with large injured liver volume and weight [58]. In our findings, the saline groups belonged to advanced 
hepatocellular carcinoma and displayed in the diffuse type, while the OCT-treated groups belonged to early hepatocellular carcinoma as expected. Moreover, degrees of liver injury were divided into negative, transforming foci, cancerous nodules, and cancer levels with increased malignancy by microscopic morphology [59]. Cells with low malignancy were rich in vessels and arranged in nested structure, while cells with high malignancy were in various sizes and shapes, mostly atypical [59-61]. Thus, the severe degree of hepatocellular carcinoma was validated by microscopic morphology and quantitated by the percentages of samples in different levels.

Long-acting OCT-PPSG demonstrated antitumor effects in both a xenograft HCC mouse model and a primary HCC rat model, which might be explained by the downregulated levels of SSTRs. As an endogenous inhibitor of secretion and cell proliferation [62], somatostatin and its analogs exerted antitumor activity through direct and indirect mechanisms by acting through SSTR1-5, which were found on most tumor cells [63]. Direct interactions with SSTRs significantly impacted tumor cell biology while indirect antitumor effects were exerted through inhibition of hormone, growth factor, and angiogenic factor secretion [64]. In the present study, high levels of SSTR2/5 in the HCC rat model were observed before treatment, which then underwent dramatic downregulation following OCT-PPSG and OCT-soln treatments. However, this could be a concern and might also be the reason for controversial therapeutic results reported in HCC patients. Somatostatin analogues (SSAs) have been reported to inhibit cell proliferation by interacting with SSTR2 and SSTR5, and the level of SSTR expression in HCC was proven to associate with the clinical outcome of LAR treatment [65], but the survival improving effects of LAR were not confirmed in a subsequent randomized placebo controlled trial. We pointed out that Sandostatin LAR monotherapy did not have survival benefit in their selected group of patients with advanced HCC [39]. Siveke JT et al reported on the complete regression of LAR in an advanced HCC patient despite recurrence of suspicious liver nodules, possibly due to tumor cells escaping SSTR treatment or downregulation of SSTR on the tumor cells [66]. The downregulation of SSTR2 levels in OCT-treated groups could result from the inhibitory effect of OCT by interacting with SSTR in tumor cells. Thus, OCT treatment might show better therapeutic response in early HCC models with high levels of SSTR expression. Additionally, previous studies found that the time of initial therapy greatly influenced antitumor effects [67, 68], which was evidenced by lower inhibitory rate when therapy began on the $7^{\text {th }}$ day after the injection of H22 hepatoma cell suspension ( $<80 \%$ by the day 12 ) than therapy began on the next day after injection ( $90 \%$ by the day 12) (SI Table 1). Regarding its clinical prospect, OCT monotherapy might not be sufficient to control and inhibit tumor progression.

Angiogenesis has been considered critical to tumor growth and progression, which is induced by a variety of growth factors such as VEGF, bFGF, and PDGF $\alpha$. VEGF has been reported to associate with tumor invasion and metastasis, and thereby, inhibition of VEGF activity and loss of its receptor (KDR) may remarkably contribute to anti-angiogenesis and antitumor effects [50]. Therefore, the level of VEGF was determined to explore the anti-angiogenesis effect of OCT.

\section{Conclusion}

In the current study, a high content $(70 \%, w / w)$ phospholipid-based phase separation gel 
loaded with octreotide (OCT-PPSG) was successfully fabricated and characterized, which could significantly prolong the in vitro release and in vivo therapeutic effect of octreotide in different animal models of three species. IVIVC study demonstrated better correlation in rabbits and Beagle dogs. The therapeutic efficacy study of OCT-PPSG was proven effective in the pretreatment of hepatocellular carcinoma in both a xenograft HCC mouse model and a primary HCC rat model. Moreover, OCT-PPSG was demonstrated to produce a stronger inhibitory effect against HCC by inhibiting VEGF secretion, tumor angiogenesis, proliferation and promoting apoptosis. Therefore, OCT-PPSG represented a promising strategy to facilitate HCC therapy. Additionally, PPSG showed great potentials for the long-term delivery of peptide and protein drugs.

\section{Acknowledgements}

This work was funded by the National Science Foundation of China (81273443), Sichuan University Startup Foundation for Talents (2082204174131) and the National S\&T Major Project of China (2014ZX09507001).

\section{References}

[1]. Lin JH, Pharmacokinetics of biotech drugs: peptides, proteins and monoclonal antibodies, Current drug metabolism. 10 (2009) 661.

[2]. Florea $\mathrm{BI}$, Thanou M, Junginger $\mathrm{HE}$, Borchard $\mathrm{G}$, Enhancement of bronchial octreotide absorption by chitosan and $\mathrm{N}$-trimethyl chitosan shows linear in vitro/in vivo correlation, Journal of Controlled Release. 110 (2006) 353-361.

[3]. Li X, Guo S, Zhu C, Zhu Q, Gan Y, Rantanen J, et al., Intestinal mucosa permeability following oral insulin delivery using core shell corona nanolipoparticles, Biomaterials. 34 (2013) 9678-9687.

[4]. Sarciaux JM, Acar L, Sado PA, Using microemulsion formulations for oral drug delivery of therapeutic peptides, International Journal of Pharmaceutics. 120 (1995) 127-136.

[5]. Mukhopadhyay P, Sarkar K, Chakraborty M, Bhattacharya S, Mishra R, Kundu PP, Oral insulin delivery by self-assembled chitosan nanoparticles: In vitro and in vivo studies in diabetic animal model, Materials Science and Engineering: C. 33 (2013) 376-382.

[6]. Chaturvedi K, Ganguly K, Nadagouda MN, Aminabhavi TM, Polymeric hydrogels for oral insulin delivery, Journal of Controlled Release. 165 (2013) 129-138.

[7]. Portero A, Remuñán-López C, Nielsen H, The Potential of Chitosan in Enhancing Peptide and Protein Absorption Across the TR146 Cell Culture Model-An in Vitro Model of the Buccal Epithelium, An Official Journal of the American Association of Pharmaceutical Scientists. 19 (2002) 169-174.

[8]. Mygind N, Dahl R, Anatomy, physiology and function of the nasal cavities in health and disease, Advanced Drug Delivery Reviews. 29 (1998) 3-12.

[9]. Zheng C, Guo Q, Wu Z, Sun L, Zhang Z, Li C, et al., Amphiphilic glycopolymer nanoparticles as vehicles for nasal delivery of peptides and proteins, European Journal of Pharmaceutical Sciences. 49 (2013) 474-482.

[10]. Kwon MJ, Bae JH, Kim JJ, Na K, Lee ES, Long acting porous microparticle for pulmonary protein delivery, International Journal of Pharmaceutics. 333 (2007) 5-9.

[11]. Patton JS, Byron PR, Inhaling medicines: delivering drugs to the body through the lungs, Nat Rev Drug Discov. 6 (2007) 67-74.

[12]. Patton JS, Platz RM, (D) Routes of delivery: Case studies: (2) Pulmonary delivery of peptides and 
proteins for systemic action, Advanced Drug Delivery Reviews. 8 (1992) 179-196.

[13]. Wan F, Møller EH, Yang M, Jørgensen L, Formulation technologies to overcome unfavorable properties of peptides and proteins for pulmonary delivery, Drug Discovery Today: Technologies. 9 (2012) e141-e146.

[14]. Pillai O, Nair V, Poduri R, Panchagnula R, Transdermal iontophoresis. Part II: Peptide and protein delivery, Methods and findings in experimental and clinical pharmacology. 21 (1999) 229.

[15]. Khafagy E-S, Morishita M, Onuki Y, Takayama K, Current challenges in non-invasive insulin delivery systems: A comparative review, Advanced Drug Delivery Reviews. 59 (2007) 1521-1546.

[16]. Lee Y-C, Simamora P, Pinsuwan S, Yalkowsky SH, Review on the systemic delivery of insulin via the ocular route, International Journal of Pharmaceutics. 233 (2002) 1-18.

[17]. Vyas SP, Paliwal R, Paliwal SR. Chapter 5 - Ocular Delivery of Peptides and Proteins. In: Walle CVD, editor. Peptide and Protein Delivery. Boston: Academic Press; 2011. p. 87-103.

[18]. de Boer AG, Moolenaar F, de Leede LGJ, Breimer DD, Rectal Drug Administration, Clin Pharmacokinet. 7 (1982) 285-311.

[19]. Mackay M, Phillips J, Hastewell J, Peptide drug delivery: Colonic and rectal absorption, Advanced Drug Delivery Reviews. 28 (1997) 253-273.

[20]. Yamamoto A, Muranishi S, Rectal drug delivery systems Improvement of rectal peptide absorption by absorption enhancers, protease inhibitors and chemical modification, Advanced Drug Delivery Reviews. 28 (1997) 275-299.

[21]. Zhang T, Peng $Q$, San F-Y, Luo J-W, Wang $M-X, W u$ W-Q, et al., A high-efficiency, low-toxicity, phospholipid-based phase separation gel for long-term delivery of peptides, Biomaterials. 45 (2015) 1-9.

[22]. Astruc B, Marbach P, Bouterfa H, Denot C, Safari M, Vitaliti A, et al., Long-acting octreotide and prolonged-release lanreotide formulations have different pharmacokinetic profiles, Journal Of Clinical Pharmacology. 45 (2005) 836-844.

[23]. Jiang $Y$, Wang J, Wang $Y$, Du X, Zhang Y, Fawcett JP, et al., Determination of long-acting release octreotide, an octapeptide analogue of somatostation, in human plasma by liquid chromatography/tandem mass spectrometry, Rapid communications in mass spectrometry : RCM. 21 (2007) 3982-3986.

[24]. Colao A, Ferone D, Lastoria S, Marzullo P, Cerbone G, DiSarno A, et al., Prediction of efficacy of octreotide therapy in patients with acromegaly, Journal of Clinical Endocrinology \& Metabolism. 81 (1996) 2356-2362.

[25]. Ambler GR, Butler AA, Padmanabhan J, Breier BH, Gluckman PD, The effects of octreotide on GH receptor and IGF-I expression in the GH-deficient rat, Journal of Endocrinology. 149 (1996) 223-231.

[26]. Sieber CC, Lee FY, Groszmann RJ, Long-term octreotide treatment prevents vascular hyporeactivity in portal-hypertensive rats, Hepatology. 23 (1996) 1218-1223.

[27]. Wang S, Preparation, Characterization and Related in Vivo Release, Safety and toxicity of Lanreotide microspheres, BiolPharmBull. 35 (2012) 1898-1906.

[28]. Hofland LJ, Lamberts SWJ, The pathophysiological consequences of somatostatin receptor internalization and resistance, Endocrine reviews. 24 (2003) 28.

[29]. Cebon J, Findlay M, Hargreaves C, Stockler M, Thompson P, Boyer M, et al., Somatostatin receptor expression, tumour response, and quality of life in patients with advanced hepatocellular carcinoma treated with long-acting octreotide, Br J Cancer. 95 (2006) 853-861.

[30]. McKeage K, Cheer S, Wagstaff A, Octreotide Long-Acting Release (LAR), Drugs. 63 (2003) 
2473-2499.

[31]. Adelman DT, Burgess A, Davies PR, Evaluation of long-acting somatostatin analog injection devices by nurses: a quantitative study, Medical devices. 5 (2012) 103-109.

[32]. Yuhua W, Determination of octreotide in the human plasma by HPLC-MS, Central South Pharmacy. 4 (2006) 174-177.

[33]. Jinsong D, Determination of octreotide in human plasma by HPLC-MS with solid-phase extraction and study on the relative bioavailability of domestic and imported octreotide injections, Acta Pharmaceutica Sinica. 39 (2004) 542-545.

[34]. STEVEN W.J. L AMBERTS AA-JAVDLE, W OUTER W. DE H ERDER , L EO J. H OFLAND Octreotide, THE NEW ENGLAND JOURNAL OF MEDICINE. 334 (1996) 246-254.

[35]. Comets E, Nonparametric analysis of the absorption profile of octreotide in rabbits from long-acting release formulation OncoLAR, Journal of Controlled Release. 59 (1999) 197-205.

[36]. Stella B, Arpicco S, Rocco F, Burgalassi S, Nicosia N, Tampucci S, et al., Nonpolymeric nanoassemblies for ocular administration of acyclovir: Pharmacokinetic evaluation in rabbits, European Journal of Pharmaceutics and Biopharmaceutics. 80 (2012) 39-45.

[37]. Yao J, Study on the determination of octreotide in beagle dogs and the bioequivalence evaluation of two long-acting release(LAR) octreotide formulations (2007).

[38]. Yang Y, Manda P, Pavurala N, Khan MA, Krishnaiah YS, Development and validation of in vitro-in vivo correlation (IVIVC) for estradiol transdermal drug delivery systems, Journal of controlled release : official journal of the Controlled Release Society. 210 (2015) 58-66.

[39]. Peilong S, Inhibition effects and mechanism studies of octreotide on HCC xenograft in mice, Chin J Hepatobiliary Surgery. 8 (2002) 305-306.

[40]. Mamot C, Drummond DC, Noble CO, Kallab V, Guo ZX, Hong KL, et al., Epidermal growth factor receptor-targeted immunoliposomes significantly enhance the efficacy of multiple anticancer drugs in vivo, Cancer Research. 65 (2005) 11631-11638.

[41]. Jia WD, Xu GL, Xu RN, Sun HC, Wang L, Yu JH, et al., Octreotide acts as an antitumor angiogenesis compound and suppresses tumor growth in nude mice bearing human hepatocellular carcinoma xenografts, Journal of cancer research and clinical oncology. 129 (2003) 327-334.

[42]. Ben-Izhak O, Laster Z, Araidy S, Nagler RM, TUNEL - an efficient prognosis predictor of salivary malignancies, Br J Cancer. 96 (2007) 1101-1106.

[43]. Denkert C, Loibl S, Mueller BM, Eidtmann H, Schmitt WD, Eiermann W, et al., Ki67 levels as predictive and prognostic parameters in pretherapeutic breast cancer core biopsies: a translational investigation in the neoadjuvant GeparTrio trial(dagger), Ann Oncol. 24 (2013) 2786-2793.

[44]. Lin M-X, Wen Z-F, Feng Z-Y, He D, Expression and significance of Bmi-1 and Ki67 in colorectal carcinoma tissues, Ai zheng = Aizheng $=$ Chinese journal of cancer. 27 (2008) 1321-1326.

[45]. Park H, Kim M, Kwon GT, Lim DY, Yu R, Sung M-K, et al., A High-Fat Diet Increases Angiogenesis, Solid Tumor Growth, and Lung Metastasis of CT26 Colon Cancer Cells in Obesity-Resistant BALB/c Mice, Molecular Carcinogenesis. 51 (2012) 869-880.

[46]. Shankar S, Marsh L, Srivastava RK, EGCG inhibits growth of human pancreatic tumors orthotopically implanted in Balb C nude mice through modulation of FKHRL1/FOXO3a and neuropilin, Molecular And Cellular Biochemistry. 372 (2013) 83-94.

[47]. Tian H, Liu S, Zhang J, Zhang S, Cheng L, Li C, et al., Enhancement of Cisplatin Sensitivity in Lung Cancer Xenografts by Liposome-Mediated Delivery of the Plasmid Expressing Small Hairpin RNA Targeting Survivin, Journal Of Biomedical Nanotechnology. 8 (2012) 633-641. 
[48]. Nakaizumi A, Uehara $H$, Baba $M$, lishi $H$, Tatsuta M, INHIBITION BY SOMATOSTATIN OF HEPATOCARCINOGENESIS INDUCED BY N-NITROSOMORPHOLINE IN SPRAGUE-DAWLEY RATS, Carcinogenesis. 14 (1993) 2601-2604.

[49]. Wang W, Xu G-L, Jia W-D, Wang Z-H, Li J-S, Ma J-L, et al., Prevention of hepatic tumor growth and metastasis in rats with rapamycin, Zhonghua gan zang bing za zhi = Zhonghua ganzangbing zazhi = Chinese journal of hepatology. 17 (2009) 193-197.

[50]. Li L-h, Antitumor and antiangiogenic activities of anti-vascular endothelial growth factor hairpin ribozyme in human hepatocellular carcinoma cell cultures and xenografts, World Journal of Gastroenterology. 13 (2007) 6425.

[51]. Ye $Q$, DepoFoam ${ }^{\mathrm{TM}}$ technology:a vehicle for controlled delivery of protein and peptide drugs, Journal of Controlled Release. 64 (2000) 155-166.

[52]. Subramanian M, Paruchury S, Singh Gautam S, Pratap Singh S, Arla R, Pahwa S, et al., Characterization of recombinantly expressed rat and monkey intestinal alkaline phosphatases: in vitro studies and in vivo correlations, Drug metabolism and disposition: the biological fate of chemicals. 41 (2013) 1425-1432.

[53]. Addeo R, Caraglia M, Del Prete S. Highlights of regional meeting of Italian Southern Oncological Group (GOIM): focus on hepatocellular carcinoma: biological and clinical background, therapeutic guide-lines and perspectives. 7 November 2008, Naples, Italy. 2009. p. 373.

[54]. Shengxue $X$, study on morphologic features during hepatocellular carcinoma induced by DEN and NMOR in rats, Journal of Hepatobiliary Surgery. 16 (2008) 135-137.

[55]. Mori W, Machinami R, Pathology of liver cirrhosis and hepatocellular carcinoma. Recent studies by Japanese workers, Acta pathologica japonica. 32 Suppl 1 (1982) 133-143.

[56]. Takayama T, Makuuchi M, Kojiro M, Lauwers GY, Adams RB, Wilson SR, et al., Early hepatocellular carcinoma: Pathology, imaging, and therapy, Annals Of Surgical Oncology. 15 (2008) 972-978.

[57]. Choi BI, Takayasu K, Han MC, SMALL HEPATOCELLULAR CARCINOMAS AND ASSOCIATED NODULAR LESIONS OF THE LIVER - PATHOLOGY, PATHOGENESIS, AND IMAGING FINDINGS, American Journal Of Roentgenology. 160 (1993) 1177-1187.

[58]. Su M. Graphic General Pathology. Beijing: Peking University Medical Press; 2005

[59]. Jayakumar S, Madankumar A, Asokkumar S, Raghunandhakumar S, Gokula dhas K, Kamaraj S, et al., Potential preventive effect of carvacrol against diethylnitrosamine-induced hepatocellular carcinoma in rats, Mol Cell Biochem. 360 (2012) 51-60.

[60]. Bennett GL, Krinsky GA, Abitbol RJ, Kim SY, Theise ND, Teperman LW, Sonographic detection of hepatocellular carcinoma and dysplastic nodules in cirrhosis: Correlation of pretransplantation sonography and liver explant pathology in 200 patients, American Journal Of Roentgenology. 179 (2002) 75-80.

[61]. Mori W, Machinami R, Tanaka K, PATHOLOGY OF HEPATOCELLULAR-CARCINOMA, Pathology Research And Practice. 169 (1980) 4-20.

[62]. Chalabi M, Duluc C, Caron P, Vezzosi D, Guillermet-Guibert J, Pyronnet S, et al., Somatostatin analogs: does pharmacology impact antitumor efficacy?, Trends in Endocrinology \& Metabolism. 25 (2014) 115-127.

[63]. Barbieri F, Bajetto A, Pattarozzi A, Gatti M, et al., Peptide Receptor Targeting in Cancer: The Somatostatin Paradigm, International Journal of Peptides. 2013 (2013) 20.

[64]. Ramirez JL, Grant M, Norman M, Wang XP, Moldovan S, de Mayo FJ, et al., Deficiency of somatostatin (SST) receptor type 5 (SSTR5) is associated with sexually dimorphic changes in the 
expression of SST and SST receptors in brain and pancreas, Molecular and cellular endocrinology. 221 (2004) 105-119.

[65]. Yao Liu, Somatostatin receptor subtypes 2 and 5 are associated with better survival in operable hepatitis B-related hepatocellular carcinoma following octreotide long-acting release treatment, Oncology letters. 6 (2013) 821-828.

[66]. Siveke JT, Herberhold C, Folwaczny C, Complete regression of advanced HCC with long acting octreotide, Gut. 52 (2003) 1531.

[67]. Anthony L, Freda PU, From somatostatin to octreotide LAR: evolution of a somatostatin analogue, Current Medical Research \& Opinion. 25 (2009) 2989-2999.

[68]. Susini C, Buscail L, Rationale for the use of somatostatin analogs as antitumor agents, Ann Oncol. 17 (2006) 1733-1742.

[69]. Nanxi Xiang, Xu Zhou, An injectable gel platform for the prolonged therapeutic effect of Pitavastatin in the management of hyperlipidemia, Journal of Pharmaceutical Sciences. (2016) 1-8. 


\section{Figure captions}

Fig. 1 Morphology of OCT-PPSG before (A and C) and after gelation (B and D). The viscosity profiles of blank PPSG and OCT-PPSG pre-gel solutions at different temperatures (E).

Fig. 2 (A) Cumulative release profiles of octreotide from OCT-PPSG in release medium with various percentages of ethanol ranging from 0 to $50 \%(\mathrm{v} / \mathrm{v}) .50 \%$ ethanol displayed the highest cumulative release. (B) The cumulative release percentage of octreotide by day 10 , which increased with the increase of ethanol content in the release medium. ${ }^{* *} p<0.01,{ }^{* * *} p$ $<0.001$. In vitro octreotide release profile from OCT-soln and OCT-PPSG in PBS (C) and 20\% EtOH (D). Data represent mean \pm SD $(n=3)$.

Fig. 3 Plasma concentration-time profiles of OCT-soln and OCT-PPSG following a single administration in rats (A), rabbits (B) and beagles (C). Data represent mean \pm SD ( $n=5)$.

Fig. 4 IVIVC model was created using Origin $^{\circledR}$ software. A second order polynomial correlation $\left(\mathrm{Y}=0.27 \mathrm{X}^{2}-3.57 \mathrm{X}+34.73\right)$ was found between the in vitro release\% $(\mathrm{X})$ and the deconvoluted (Fit) in vivo absorption\% (Y) with a correlation coefficient $R^{2}$ of 0.9488 , indicating a relative strong correlation between the two components. The in vitro release experiment was conducted using $20 \%(\mathrm{v} / \mathrm{v})$ ethanol in the release medium (A). A second order polynomial correlation $\left(\mathrm{Y}=0.05 \mathrm{X}^{2}-0.51 \mathrm{X}+29.27\right.$ ) was found between the in vitro release\% $(\mathrm{X})$ and the deconvoluted (Fit) in vivo absorption\% (Y) with a correlation coefficient $R^{2}$ of 0.8534 , indicating a relative weak correlation between the two components. The in vitro release experiment was conducted using PBS in the release medium (B). Manually calculated in vivo absorption\% based on the label information was also shown at each time point for comparison (square).

Fig. 5 (A) Images of $\mathrm{H} 22$ tumors excised from the respective treatment groups after the $14^{\text {th }}$ day in H22-bearing mice. Scale bar $=1 \mathrm{~cm}$. (B) The change of tumor volume of the respective treatments in H22-bearing mice. (C) Body weight (g) of each treatment group measured on day 0 and 14 indicating no significant differences across different treatment groups. (D) Tumor inhibitory rate for OCT-soln and OCT-PPSG on day 7, 10, 12, 14 as calculated by the percentage of tumor volume reduction. Saline only was accepted as the control group, the inhibitory rate of which was considered as zero. (E) Survival curve.

Fig. 6 (A) Hematoxylin and eosin (H\&E) staining of tumor sections collected from different treatment groups. Photomicrographs were taken with a microscope camera at 40× magnification while representative areas were enlarged at 200× magnification. (B) Immunohistological staining for CD31, Ki-67 and TUNEL. Photomicrographs were taken with a microscope camera at $200 \times$ magnification. Scale bar $=50 \mu \mathrm{m}$.

Fig. 7 (A) Method outlines to establish primary hepatocellular carcinoma model in rats. Macroscopic gross appearance of livers at the end of experimental period in control and experimental group of rats (Scale bar $=5 \mathrm{~mm}$ ). a Liver of a control animal showing normal 
morphology. b DEN and NMOR + saline group showing heavy enlargement and several grayish white nodules and foci on the peripheral surface of the liver as the features of HCC. $\mathbf{c}$ DEN and NMOR + OCT-soln group showing most of the foci and nodules disappeared in the liver. d DEN and NMOR + OCT-PPSG group showing most of the foci and nodules disappeared in the liver. Figures below are representative images of liver tissue sections stained with hematoxylin and eosin (H\&E). Images were taken with a microscope camera at $200 \times$ magnification (Scale bar $=50 \mu \mathrm{m}$ ). (B) Levels of VEGF in serum of control and different experimental groups determined by ELISA. Data represent mean \pm SD $(n=10)$ for each condition. ${ }^{*} p<0.05$ vs saline group; ${ }^{* *} p<0.01$ vs saline group. (C) Immunohistological staining of SSTR2 and SSTR5 in liver sections. Images were taken with a microscope camera at $400 \times$ magnification (Scale bar $=50 \mu \mathrm{m}$ ). (D) Levels of SSTR2 and SSTR5 expression in rats with primary hepatocellular carcinoma following pretreatment with saline, OCT-soln or OCT-PPSG. Data represent mean \pm SD of five animals per each condition. 